\title{
COMBINED PERCUTANEOUS RETROGRADE AORTIC AND TRANSSEPTAL LEFT HEART CATHETERIZATION*
}

\author{
BY \\ G. B. PECKHAM, A. CHRYSOHOU, H. E. ALDRIDGE, AND E. D. WIGLE \\ From the Department of Medicine, University of Toronto and the Cardiovascular Unit, Toronto General Hospital, \\ Toronto, Canada
}

Received September 20, 1963

Left heart catheterization in man has assumed increasing importance with the rapid advances in cardiac surgery for lesions of the left side of the heart. Zimmerman, Scott, and Becker (1950) were the first to perform left ventricular catheterization, passing a catheter in retrograde fashion from aorta to left ventricle. Subsequently the transbronchial (Facquet et al., 1952; Allison and Linden, 1953), transthoracic (Björk, Malmström, and Uggla, 1953), suprasternal (Radner, 1954), and direct ventricular puncture (Brock, Milstein, and Ross, 1956) techniques have been used widely; but each has certain disadvantages (Morrow, Braunwald, and Ross, 1960). Ross (1959) and Cope (1959) almost simultaneously described the transseptal technique of left heart catheterization. Cope (1959) was the first to apply this technique in man. Ross, Braunwald, and Morrow (1960) have described the original transseptal technique in detail. Subsequently the improved and modified transseptal technique of Brockenbrough, Braunwald, and Ross (1962) has been described and has gained wide acceptance. This report describes our experience in combining this improved transseptal technique with the retrograde technique of left ventricular catheterization. This represents a combination of the newest and oldest methods of left heart catheterization, a combination that permits a complete assessment of both stenosis and regurgitation at aortic and mitral valves, including hæmodynamic, angiographic, and dye dilution studies, and overcomes some of the limitations encountered using either technique alone. Right heart catheterization may be combined easily with this procedure. One of the distinct advantages is the fact that both the transseptal and retrograde catheters can be introduced percutaneously from the right groin. McIntosh et al. (1961) have previously reported the combination of retrograde left ventricular catheterization with the original Ross technique of transseptal left atrial catheterization for the assessment of mitral valve disease. The combination of techniques described here is particularly suited for the assessment of lesions obstructing left ventricular outflow, as well as for the assessment of mitral valve disease.

\section{The Combined Technique}

Percutaneous Retrograde Aortic Catheterization. After local anæsthetic has been injected into the right groin, a retrograde aortic catheterf is introduced into the right femoral artery by the catheter replacement technique (Seldinger, 1953). This catheter is then advanced to the ascending aorta and its tip positioned at the aortic valve. This part of the procedure is performed first, as this catheter then accurately locates the aorta for reference during transseptal left heart catheterization.

* Supported by the Ontario Heart Foundation and the Canadian Life Insurance Medical Fellowship.

$\dagger$ No. 1 or No. 3 Odman-Ledin, Kifa, Solna 3, Sweden, prepared with tapered end and four side holes in the distal $2 \mathrm{~cm}$. of the catheter. 
Percutaneous Transseptal Left Heart Catheterization. This technique has been described in detail (Brockenbrough et al., 1962), and reference will be made here only to several small but, in our hands, helpful modifications. A spring guide wire, having been introduced into the femoral vein percutaneously (Cope 1959; Brockenbrough et al., 1962), is advanced directly to the right atrium, and used as the guide for the introduction of the "teflon" transseptal catheter into that chamber. By so doing, the need for a steel guide for the introduction of the transseptal catheter into the right atrium (Brockenbrough et al., 1962) has been abolished and the procedure shortened by one step. Sometimes there has been considerable resistance to the introduction of the Brockenbrough "teflon" catheter over the spring guide wire through the wall of the femoral vein, and the curve at the catheter tip has been lost. This difficulty has been overcome by first introducing into the femoral vein, over the spring guide wire, a short length of No. 1 OdmanLedin catheter with tapered tip, which is then withdrawn. The entry into the femoral vein made with this Odman-Ledin catheter facilitates the intro-

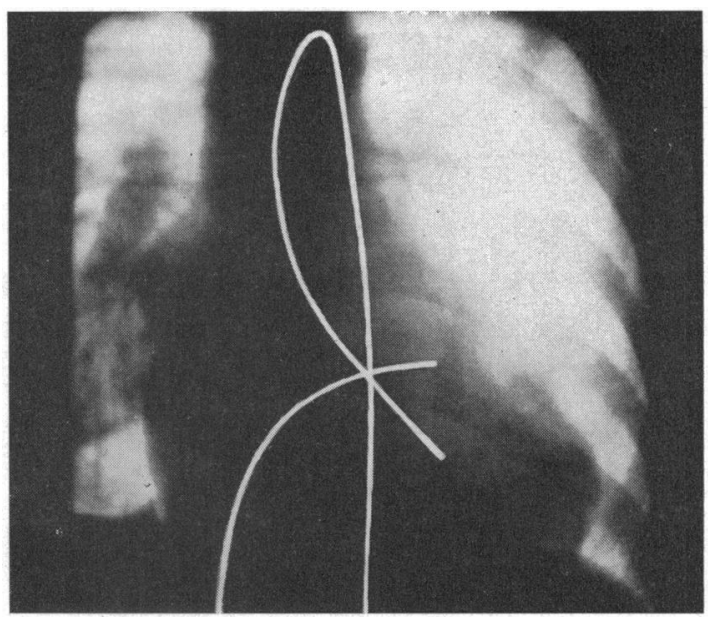

FIG. 1.-Radiograph showing the retrograde catheter positioned in the left ventricle and the transseptal catheter positioned in the left atrium for the assessment of mitral valve disease (see Fig. 2). The catheters have been retouched for clarity of illustration in this figure and in Fig. 4. duction of the "teflon" transseptal catheter.

Once the transseptal catheter is in the right atrium, the spring wire is withdrawn, the transseptal needle is introduced, the interatrial septum is punctured, and the transseptal catheter is positioned in the left atrium (Brockenbrough et al., 1962).

Assessment of Mitral Valve Disease. (a) When it is possible to advance the retrograde aortic catheter into the left ventricle, the transseptal catheter is positioned in the left atrium (Fig. 1). With the catheters in these respective positions simultaneous left atrial and ventricular pressure tracings may be recorded for the assessment of mitral stenosis (Fig. 2A). The estimation of the degree of mitral insufficiency by dye dilution techniques is also readily performed by injecting the indicator dye into the left ventricle via the retrograde catheter. The regurgitant dye is sampled by withdrawal of blood from the left atrium via the transseptal catheter, the forward flow of dye being recorded by an oximeter on the right ear (Fig. 2B). Left ventricular cineangiography, to demonstrate mitral insufficiency, can be performed using the retrograde catheter in the left ventricle to inject the opaque media. By using the retrograde catheter, rather than the transseptal catheter positioned in the left ventricle, the possibility that the latter may at times distort the mitral leaflets and produce artefactual insufficiency is avoided.

Sometimes, in the presence of a giant left atrium or severe mitral stenosis, it is difficult or impossible to advance the transseptal catheter into the left ventricle, and in these cases the use of the combined technique avoids the necessity for introducing the transseptal catheter into the left ventricle.

(b) When it is not possible to introduce the retrograde aortic catheter into the left ventricle, the transseptal catheter may be used. To measure simultaneous left atrial and ventricular pressures under these conditions polyethylene tubing has been introduced through the transseptal catheter into the left ventricle, the transseptal catheter then being withdrawn to the left atrium, leaving the polyethylene tubing in the ventricle (Brockenbrough et al., 1962). We found that polyethylene tubing was unsatisfactory for this purpose as (a) it was difficult to introduce because of its pliable nature, (b) dye dilution estimation of mitral insufficiency was not possible, and (c) the simultaneous left atrial and ventricular pressure curves were frequently too damped to be meaningful. These difficulties were overcome by using nylon tubing* in place of the polyethylene. The nylon tubing, being stiffer, could be more easily introduced through the transseptal catheter. Simultaneous left atrial and ventricular pressures were obtainable for the assessment of mitral stenosis (Fig. 3A), and dye curve estimation of mitral insufficiency was possible (Fig. 3B). In this latter instance the indicator dye was injected into the left ventricle via the nylon tubing, the regurgitant dye was sampled

* Outer diameter 0.037 in., internal diameter 0.019 in. United States Catheter and instrument Corporation, Glen Falls, N.Y. 
by the withdrawal of blood from the left atrium via the transseptal catheter, and the forward flow of dye was recorded by an oximeter on the right ear.

Using this combined technique, and thus having these two alternative ways by which mitral valve disease

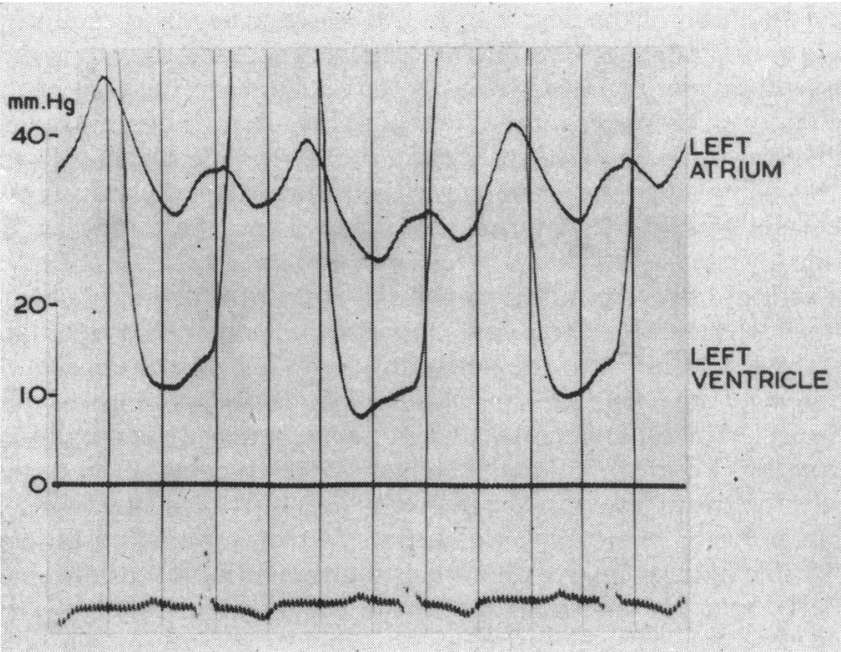

(A)

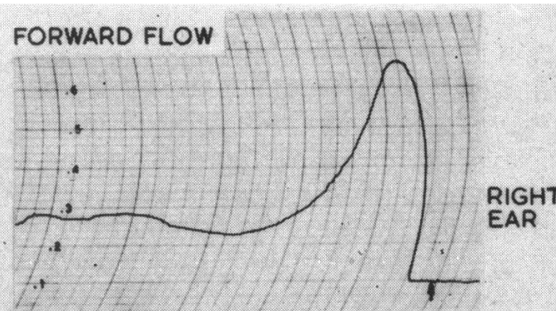

REGURGITANT FLOW

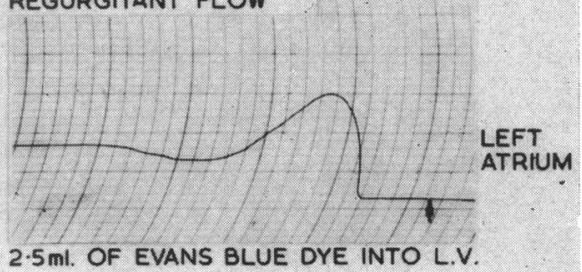

(B)

Fig. 3.-Assessment of mitral valve disease with the inner nylon tubing positioned in the left ventricle and the transseptal catheter situated in the left atrium (see text). (A) Simultaneous left atrial and ventricular pressure traces from a case of mitral stenosis and insufficiency. (B) Dye dilution estimation of mitral insufficiency from the same case. The Evans Blue dye was injected into the left ventricle via the nylon tubing, the regurgitant dye being sampled via the transseptal catheter in the left atrium. Forward flow was recorded by an oximeter on the right ear. 


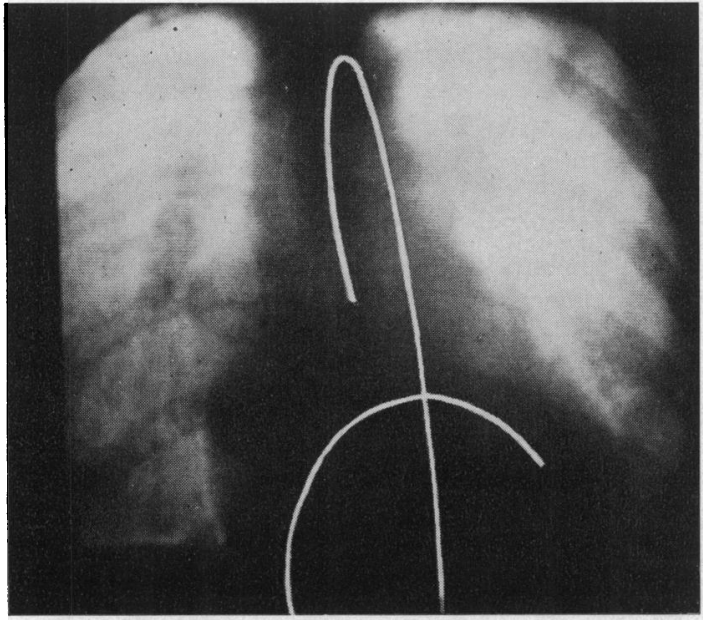

FIG. 4.-Radiograph showing the retrograde catheter positioned above the aortic valve and the transseptal catheter positioned in the left ventricle for hæmodynamic, dye dilution, and angiographic assessment of aortic valve disease.

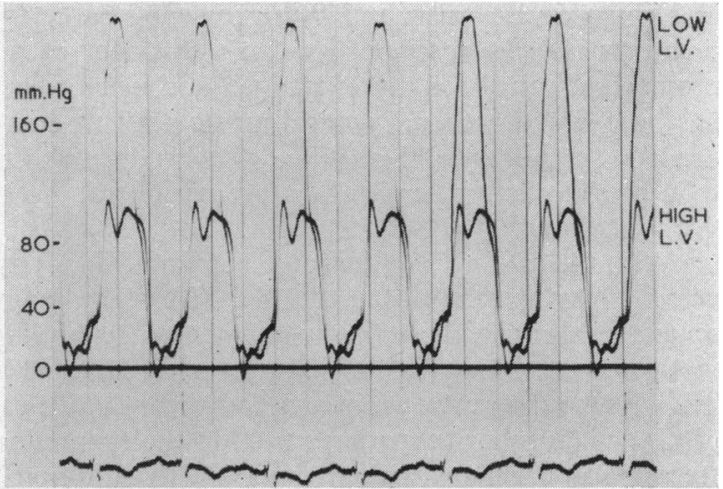

Fig. 6.-Simultaneous low and high left ventricular pressures recorded via the transseptal and retrograde catheters respectively, in a case of discrete sub-aortic stenosis. The combined technique is particularly suited for such a case, in that by injection of indicator dye or radio-opaque substance into the aortic root via the retrograde catheter the degree of aortic insufficiency may be estimated by dye dilution or angiographic means, whereas a left ventricular angiogram via the transseptal catheter allows visualization of the left ventricular outflow tract.

can be assessed, has been found convenient. Positioning the retrograde catheter in the left ventricle, with the transseptal catheter in the left atrium, facilitates the accurate assessment of mitral valve disease. Only when it was not possible to introduce the retrograde catheter into the left ventricle, as in cases of aortic stenosis, has the nylon tubing within the transseptal catheter been used for the assessment of mitral disease.

Assessment of Aortic Disease. The combined catheterization technique is probably even better suited for the assessment of lesions of the left ventricular outflow tract. With the retrograde catheter positioned

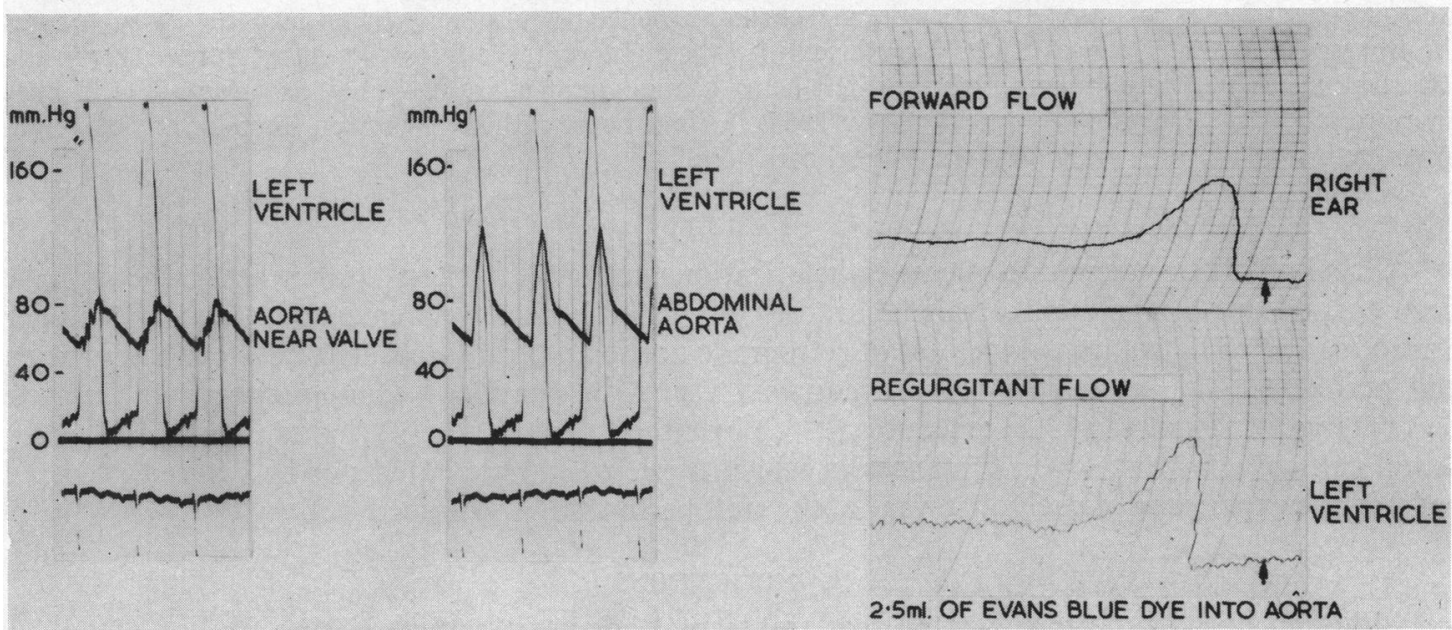

(A)

(B)

FIG. 5.-Assessment of aortic valve disease with the retrograde aortic catheter positioned in the aortic root and the transseptal catheter positioned in the left ventricle (see Fig. 4). (A) Simultaneous left ventricular and aortic pressure traces from a case of aortic stenosis and insufficiency. Note that in the presence of aortic insufficiency the systolic and pulse pressures in the aorta increase as the retrograde catheter is withdrawn from the region of the aortic valve (see text). (B) Dye dilution estimation of aortic insufficiency in same case. The Evans Blue dye was introduced into the aorta via the retrograde catheter, the regurgitant dye sampled from the left ventricle via the transseptal catheter and forward flow of dye was recorded by an oximeter on the right ear. 
above the aortic valve and the transseptal catheter positioned in the left ventricle (Fig. 4) simultaneous pressures may be recorded for the assessment of the degree of aortic stenosis present (Fig. 5A). In the presence of combined aortic stenosis and insufficiency, the systolic pressure gradient across the aortic valve has been greater when a central aortic pressure was recorded than when the arterial pressure has been recorded at a more peripheral site. This is due to the fact that in the presence of aortic insufficiency, the systolic pressure is greater in the more peripheral arterial tracings (Fig. 5A), as described by Remington and Wood (1956).

With the catheters in the same position (Fig. 4) dye dilution evidence of the degree of aortic insufficiency present may also be obtained (Fig. 5B). The indicator dye is injected into the aortic root, over several cardiac cycles, via the retrograde catheter, regurgitant dye is sampled from the left ventricle via the transseptal catheter, forward-flowing dye being sampled by an ear oximeter. By comparing the area under the dye curves representing forward and regurgitant flow, an estimate of the degree of aortic insufficiency can be obtained similar to methods used in the estimation of mitral insufficiency (Gorelick et al., 1962). When comparison has been made between the dye dilution and cineangiographic estimation of aortic insufficiency, there has been close agreement in the two estimates of the severity of the lesion. When such comparisons have been made, care has been taken to have the aortic catheter in the same position for both the injection of dye and the injection of the radio-opaque material for the angiogram, i.e. 2 to $3 \mathrm{~cm}$. above the aortic valve.

The aortic cineangiogram, in addition to demonstrating the degree of aortic insufficiency present, in a high percentage of cases outlined the coronary arterial tree in a satisfactory manner. Such visualization of the coronary circulation in cases of aortic valve disease is often important in the assessment of such cases before cardiac surgery.

Using this combined technique in the assessment of subaortic stenosis, simultaneous high and low left ventricular pressures may be recorded via the retrograde and transseptal catheters respectively (Fig. 6). In the case (Fig. 6) of discrete sub-aortic stenosis, the degree of aortic insufficiency present was estimated by dye dilution studies (see above) and by aortic cineangiography via the retrograde catheter. A left ventricular cineangiogram, via the transseptal catheter, outlined the anatomy of the left ventricular outflow tract. In such cases the retrograde aortic catheter may also be used to obtain a withdrawal pressure tracing from low left ventricle to aorta.

Right heart catheterization may be carried out before (Brockenbrough et al., 1962), during, or after the left heart studies. In cases of valvular disease of the left heart, it is frequently sufficient to record right atrial and ventricular pressures to assess the presence or absence of pulmonary hypertension. In such cases the transseptal catheter can easily be introduced into the right ventricle following its withdrawal from the left atrium. When simultaneous right and left heart hæmodynamics are desirable, as during drug studies or exercise (Wigle, Chrysohou, and Bigelow, 1963), a right heart catheter has been introduced before the introduction of the retrograde and transseptal catheters.

\section{RESULTS}

A total of 158 transseptal left heart catheterizations, using the "teflon" transseptal catheter, have been carried out by the staff of this laboratory. The left atrium was entered in 153 or in 97 per cent of cases. The combined method of retrograde aortic and transseptal left heart catheterization was performed in 142 of these 158 procedures. Using the combined technique, the left ventricle was entered in 132 of the 142 procedures $(93 \%)$ by either, or both, the retrograde aortic and transseptal catheters. Most of the instances in which the left ventricle could not be entered occurred early in the experience of each of several of the staff performing the procedures.

\section{COMPLiCATIONS}

Although initial reports suggested that transseptal left heart catheterization was an extremely safe procedure (Ross et al., 1960; Brockenbrough et al., 1962), the complications listed below indicate that there are hazards in the use of this technique as with other techniques of left heart catheterization.

Aortic Puncture. There were two instances in which the root of the aorta was entered inadvertently during transseptal left heart catheterization. In the first instance only the needle tip entered this vessel. The patient had a pericardial friction rub for several days following the pro- 
cedure, but pericardial tamponade did not occur. The second instance of aortic root puncture occurred in a cachectic (height $5 \mathrm{ft} .6 \mathrm{in} .(167 \mathrm{~cm}$.), weight $83 \mathrm{lb}$. $(37 \cdot 6 \mathrm{~kg}$.)) 58-year-old man with mitral stenosis. In this instance, the "teflon" catheter as well as the needle entered the aortic root, and cardiac tamponade ensued. The pericardium was drained by operation, but the patient died suddenly one-and-a-half hours later. At necropsy there was severe mitral stenosis, complete old thrombotic occlusion of the left main pulmonary artery, and the site of aortic puncture could be identified.

These two instances of aortic root puncture occurred in patients with moderate or great enlargement of the left and right atria and they raise several points in technique, for example the importance of identifying the chamber entered by the transseptal needle (Brockenbrough et al., 1962), by obtaining oxygenated blood and recording pressures, before advancing the catheter along with the needle into the chamber. In the first instance of aortic puncture, only the needle tip entered the aorta and there were no adverse effects. In the second instance, the catheter entered this vessel and resulted in cardiac tamponade and death. In this latter case the catheter penetrated the aorta while the pressure was being recorded through the needle to identify the chamber entered. The reason for this happening could possibly be related to the cachectic state of the patient or may have been related to the fact that "teflon" catheters lengthen with time. When lengthening of the catheter occurs, the transseptal needle protrudes less from its tip. Hence, when needle puncture occurs, there is greater likelihood that the tip of the catheter may also penetrate. Since this episode, where the needle only protruded $4 \mathrm{~mm}$. from the catheter tip, instead of the usual $10 \mathrm{~mm}$., catheters have been shortened so that the needle always protrudes at least $8 \mathrm{~mm}$. This tends to ensure needle entry alone with the initial puncture.

Having the retrograde aortic catheter placed in the aorta was helpful in locating this vessel, and in avoiding puncture of it, during transseptal left heart catheterization. In several patients with moderate or markedly enlarged left and right atria, in whom the transseptal catheter and needle continually slipped over the septal wall and up to the region of the aorta, it was felt that having the retrograde catheter in the aorta possibly prevented mistaken puncture of that vessel. On the other hand, in cases of aortic valve disease, with normal or slightly enlarged left atria, having the tip of the aortic catheter positioned at the aortic valve, has demonstrated that puncture of the interatrial septum by the transseptal needle and catheter frequently occurred 3 to $4 \mathrm{~cm}$. above the plane of the aortic valve. In these instances the septal puncture occurred above the lower one-half of the lateral right atrial silhouette, the suggested region for puncture of the septum (Ross et al., 1960). It is known in a number of such instances that this higher location of the puncture was correct (i.e. the upper border of the fossa ovalis), because the catheter and needle slipped through the foramen ovale together without having to protrude the needle for septal puncture. (One of us has entered the left atrium in 40 of 50 transseptal left heart catheterizations without having to protrude the needle beyond the catheter tip, by exploring the septum until the upper rim of the fossa ovalis is encountered.) With gentle pressure the catheter will then enter the left atrium (H. E. Aldridge, unpublished observations).

Death Following Procedure. One patient died suddenly two days following the procedure. This 56-year-old man experienced ischæmic chest pain at the time of puncture of the interatrial septum. This passed off, the procedure was completed, and the patient was comfortable the following day. Death occurred suddenly 48 hours after the procedure. Necropsy revealed severe aortic stenosis, severe coronary artery disease with complete sclerotic occlusion of the right coronary artery, and a normally situated septal puncture (upper rim of fossa ovalis).

Puncture of the right atrial wall by the "teflon" catheter occurred in two instances but had no ill effect on the patients.

Puncture of the left atrial wall occurred once early in our experience: in this case the curve of the "teflon" transseptal catheter was lost because of difficulty in introducing the catheter over the spring guide wire into the femoral vein. As a result the catheter tip in the left atrium did not curve inferiorly once the needle was withdrawn but pointed to the lateral left atrial wall, and in trying to 
move the catheter along this wall it entered the pericardium. Tamponade ensued, the pericardium was drained, and the patient subsequently underwent successful mitral commissurotomy.

To prevent such distortion of the curve at the end of the transseptal catheter, the small No. 1 Odman-Ledin catheter has been first introduced into the femoral vein over the spring guide and then withdrawn to allow subsequent easier entry of the transseptal catheter over the spring wire into the vein. In addition, if a transseptal catheter in the left atrium points laterally and to the left and will not direct itself inferiorly for entry into the left ventricle, such a catheter is replaced. This change of catheters has been accomplished on several occasions by introducing the spring guide wire into the left atrium through the original catheter. This catheter is then withdrawn, leaving the guide wire in the left atrium. The new transseptal catheter can then be threaded over the spring wire directly into the left atrium, thus avoiding a second puncture of the interatrial septum.

Femoral Artery Thrombosis. There were three instances of thrombosis of the femoral artery following percutaneous retrograde aortic catheterization via that vessel. This complication only occurred in small thin women, when the large No. 3 Odman-Ledin catheter was used. Subsequently the smaller No. 1 Odman-Ledin catheter has been used in people of small build and no further thromboses have occurred. In each case where a thrombosis did occur, it was removed successfully under local anæsthesia.

Systemic Emboli Following the Procedure. There have been three instances of systemic emboli (one cerebral, one splenic, and one renal) within 48 hours of transseptal left heart catheterization. In each case, the effects of the embolus were transient. It is not known whether these emboli arose from the site of septal puncture or not. Thrombosis on the left atrial wall, at the site of septal puncture, has recently been reported (Pinkerson, Kelser, and Adkins, 1963).

\section{Discussion}

The advantages of percutaneous transseptal left heart catheterization using the "teflon" catheters have been outlined (Brockenbrough et al., 1962) as have the advantages of percutaneous retrograde aortic and left ventricular catheterization (Dotter, 1960). The use of these two established techniques in combination, as described in this report, appears to offer further advantages that are not available using either technique alone. At the same time, the use of this combined technique overcomes disadvantages inherent in the use of either the transseptal or retrograde techniques alone.

In the assessment of mitral valve disease, positioning of the retrograde catheter in the left ventricle and the transseptal catheter in the left atrium allows hæmodynamic, angiographic, and dye dilution estimation of the degree of stenosis and/or insufficiency present. In the presence of a giant left atrium or severe mitral stenosis, it can be difficult, and at times impossible, to introduce the "teflon" transseptal catheter into the left ventricle. By introducing the retrograde catheter into that chamber, this difficulty can be circumvented. Similarly, it is not necessary to use polyethylene or nylon tubing within the transseptal catheter to record simultaneous left atrial and ventricular pressure when the retrograde catheter can be positioned in the left ventricle. Dye dilution estimation of the degree of mitral insufficiency present is also facilitated by the combined technique (Fig. 2B).

This combined technique is probably even more advantageous in the study of disease of the left ventricular outflow tract. With the retrograde catheter situated in the ascending aorta and the transseptal catheter in the left ventricle, complete hæmodynamic, angiographic, and dye dilution assessment of aortic stenosis and insufficiency is possible. Aortic angiography via the retrograde catheter allows visualization of the degree of aortic insufficiency, of the coronary arterial tree, and of the anatomy of the aortic valve and ascending aorta. Left ventricular angiography via the transseptal catheter allows visualization of the left ventricular outflow tract.

Both the transseptal and retrograde catheters are introduced percutaneously from the right groin making catheter manipulation both easy and localized. Right heart assessment can be carried out before, during, or after left heart assessment. The complete hæmodynamic, angiographic, and dye dilution assessment permitted by this combined transseptal and retrograde technique avoids the 
necessity of multiple cardiac catheterizations in the investigation of any one patient. Previously in this laboratory, to obtain all the information possible by using this combined technique, three types of heart catheterization would have been required; right heart catheterization, transthoracic left heart and retrograde aortic catheterization. Thus, this combined procedure results in an economy of time for patient and doctor alike.

The complications encountered during transseptal left heart catheterization in this series of cases indicate that this procedure is not without risks, although in early reports (Ross et al., 1960; Brockenbrough et al., 1962) no serious complications were encountered. Recently, Adrouny et al. (1963) have stressed the danger of puncturing the wall of the heart during this procedure. This complication occurred in 9 of 191 consecutive transseptal left heart catheterizations (Adrouny et al., 1963). The present report and that of Adrouny et al., serve to create further awareness of the potential hazards of this extremely useful and widely accepted form of left heart catheterization.

\section{SUMMARY}

Experience with 142 combined percutaneous retrograde aortic and transseptal left heart catheterizations has been presented. The advantages of this combination of the oldest and newest techniques of left heart catheterization are: (1) both catheters are introduced percutaneously from the right groin; (2) it allows a more complete hæmodynamic, angiographic, and dye dilution assessment of left heart lesions than was previously possible during one catheterization procedure; (3) it can readily be combined with right heart catheterization, before, during, or after the left heart assessment; and (4) it avoids the need for multiple types of heart catheterization, thus saving time.

The major risk of the procedure in our experience is that of aortic root puncture by the transseptal needle and catheter. This danger existed almost entirely in patients with gross left and right atrial enlargement: in these, the transseptal catheter appeared to slip up over the atrial septum to the region of the aortic root. Having the retrograde aortic catheter positioned in the root of the aorta helped to identify this vessel, and on occasion it was felt to have helped in avoiding mistaken aortic root puncture.

\section{ADDENDUM}

Recently Armelin et al. (1963) have demonstrated that, in dogs, the detection and measurement of experimentally produced aortic regurgitation by means of indicator-dilution curves recorded from the left ventricle is an accurate means of assessment of this lesion. The combined catheterization technique described herein is eminently suited for such estimation of aortic regurgitation in man (see above).

The authors would like to thank Dr. W. F. Greenwood for his interest and encouragement in this work, to acknowledge the work of Drs. Donald Beanlands, John Morch, and John Spears in performing the procedures, and to thank Miss Jean McMeekan, Mrs. Erin Holly, Mrs. Renate Kaiser, Mrs. Anne Kacic, and Mr. Toivo Nelberg for technical assistance, and Miss Carole Wilkinson for secretarial assistance.

\section{REFERENCES}

Adrouny, Z. A., Sutherland, D. W., Griswold, H. E., and Ritzmann, L. W. (1963). Complications with transseptal left heart catheterization. Amer. Heart J., 65, 327.

Allison, P. R., and Linden, R. J. (1953). The bronchoscopic measurement of left auricular pressure. Circulation, 7, 669.

Armelin, E., Michaels, L., Marshall, H. W., Donald, D. E., Cheesman, R. J., and Wood, E. H. (1963). Detection and measurement of experimentally produced aortic regurgitation by means of indicator-dilution curves recorded from the left ventricle. Circulat. Res., 12, 269.

Björk, V. O., Malmström, G., and Uggla, L. G. (1953). Left auricular pressure measurements in man. Ann. Surg., $138,718$.

Brock, R., Milstein, B. B., and Ross, D. N. (1956). Percutaneous left ventricular puncture in the assessment of aortic stenosis. Thorax, 11, 163.

Brockenbrough, E. C., Braunwald, E., and Ross, J., Jr. (1962). Transseptal left heart catheterization. A review of 450 studies and description of an improved technic. Circulation, 25, 15. 
Cope, C. (1959). Technique for transseptal catheterization of the left atrium: Preliminary report. J. thorac. Surg., $37,482$.

Dotter, C. T. (1960). Left ventricular and systemic arterial catheterization: A simple percutaneous method using a spring guide. Amer. J. Roentgenol., 83, 969.

Facquet, J., Lemoine, J. M., Alhomme, P., and Lefebvre, J. (1952). La mesure de la pression auriculaire gauche par voie transbronchique. Arch. Mal. Caur, 45, 741.

Gorelick, M. M., Lenkei, S. C. M., Heimbecker, R. O., and Gunton, R. W. (1962). Estimation of mitral regurgitation by injection of dye into the left ventricle with simultaneous left atrial sampling. Amer. J. Cardiol., $10,62$.

McIntosh, H. D., Sleeper, J. C., Thompson, H. K., and Whalen, R. E. (1961). Simplification of left heart catheterization percutaneous techniques for catheter insertions. J. Amer. med. Ass., 177, 600 .

Morrow, A. G., Braunwald, E., and Ross, J., Jr. (1960). Left heart catheterization. Arch. intern. Med., $105,645$.

Pinkerson, A. L., Kelser, G. A., Jr., and Adkins, D. C. (1963). Mural thrombus in the left atrium secondary to transseptal catheterization of the left side of the heart. New Engl. J. Med., 268, 367.

Radner, S. (1954). Suprasternal puncture of the left atrium for flow studies. Acta med. scand., $148,57$.

Remington, J. W., and Wood, E. H. (1956). Formation of peripheral pulse contour in man. J. appl. Physiol., 9, 433.

Ross, J., Jr. (1959). Transseptal left heart catheterization: A new method of left atrial puncture. Ann. Surg., 149, 395.

—- Braunwald, E., and Morrow, A. G. (1960). Left heart catheterization by the transseptal route: A description of the technic and its applications. Circulation, 22, 927.

Seldinger, S. I. (1953). Catheter replacement of the needle in percutaneous arteriography. A new technique. Acta radiol. (Stockh.), 39, 368.

Wigle, E. D., Chrysohou, A., and Bigelow, W. G. (1963). Results of ventriculomyotomy in muscular subaortic stenosis. Amer. J. Cardiol., 11, 572.

Zimmerman, H. A., Scott, R. W., and Becker, N. O. (1950). Catheterization of the left side of the heart in man. Circulation, 1, 357. 